\title{
Study of the Effect of Relativistic Time Dilation on Cosmic Ray Muon Flux - Undergraduate Modern Physics Experiment
}

Nalini Easwar

Smith College, neaswar@smith.edu

Douglas A. Maclntire

Smith College

Follow this and additional works at: https://scholarworks.smith.edu/phy_facpubs

Part of the Physics Commons

\section{Recommended Citation}

Easwar, Nalini and Maclntire, Douglas A., "Study of the Effect of Relativistic Time Dilation on Cosmic Ray Muon Flux - Undergraduate Modern Physics Experiment" (1991). Physics: Faculty Publications, Smith College, Northampton, MA.

https://scholarworks.smith.edu/phy_facpubs/41

This Article has been accepted for inclusion in Physics: Faculty Publications by an authorized administrator of Smith ScholarWorks. For more information, please contact scholarworks@smith.edu 


\title{
Study of the effect of relativistic time dilation on cosmic ray muon flux-An undergraduate modern physics experiment
}

\author{
Nalini Easwar and Douglas A. Maclntire ${ }^{\text {a) }}$ \\ Department of Physics, Smith College, Northampton, Massachusetts 01063
}

(Received 27 February 1990; accepted for publication 24 November 1990)

\begin{abstract}
An experiment to study the effect of relativistic time dilation on secondary muon fluxes observed at different altitudes is described in this article. Muons, produced as secondary particles from the interaction of primary cosmic rays with the upper atmosphere, form a natural and abundant source of subatomic "clocks" moving at very high speeds. The measured muon flux on a mountain relative to that measured at sea level can be compared to predictions from calculations that take into account the relativistic time dilation in the muon frame. Situations under which such an experiment can be successfully performed are explored with a day-long field trip to a nearby mountain. This experiment has been developed at Smith College as a module in the Five College cooperative undergraduate advanced laboratory course (other participating institutions are Amherst College, Mount Holyoke College, and the University of Massachusetts).
\end{abstract}

\section{INTRODUCTION}

The muon is unstable and spontaneously decays to an electron according to

$$
\mu^{-} \rightarrow e^{-}+v_{\mu}+\bar{v}_{e}, \mu^{+} \rightarrow e^{+}+\bar{v}_{\mu}+v_{e},
$$

with a lifetime $\tau$ of approximately $2.2 \mu \mathrm{s}$. As the muons decay in flight, the measured flux $N_{s}$ at sea level is lower than the measured flux $N_{m}$ on a mountain, given by $N_{s}=N_{m} \exp (-t / \tau)$, where $t$ is the travel time from the mountain to sea level. The effect of time dilation in the muon frame keeps them "younger" and the actual flux at sea level is higher than that expected from a nonrelativistic calculation and is given by

$$
N_{s}=N_{m} \exp \left[-t_{\mu} / \tau\right]=N_{m} \exp \left[-t_{E} /(\gamma \tau)\right],
$$

where $t_{\mu}$ is the elapsed time in the moving frame of the muon, $t_{E}$ is the elapsed time in the Earth frame, $\gamma=1 /\left(1-v^{2} / c^{2}\right)^{1 / 2}$, and $v$ is the velocity of the muons in the Earth's frame. Knowing the height of the mountain and the velocity of the muons, the measured fluxes can be easily compared with the calculations.

During the years 1939-1943, several experiments were done by Rossi and co-workers ${ }^{1}$ to study the absorption of mesons in different substances, the mean lifetime of decay of the mesons, and the effects of relativistic time dilation in the frame of the mesons in flight. A later experiment by Frisch and Smith $^{2}$ demonstrated time dilation using muon fluxes measured on Mt. Washington, New Hampshire (altitude of $1910 \mathrm{~m}$ ) and at Cambridge, MA ( $3 \mathrm{~m}$ above sea level). The experiment was the basis for a film often shown in introductory modern physics courses: "Time dilation: An experiment with $\mu$ mesons." In this experiment the detector was placed under a layer of iron approximately 0.75 m thick. This arrangement sampled muons moving at speeds between $0.9950 \mathrm{c}$ and $0.9954 \mathrm{c}$. The advantage of using high-velocity muons was twofold: (i) the relativistic time-dilation effect is greater and (ii) the fractional energy loss of the muons in the atmosphere is negligible, making it a constant velocity problem. On the other hand, the disadvantage of transporting such large amounts of lead or iron is evident.

The work reported here demonstrates the feasibility of an experiment with the following three goals: (i) minimiz- ing the amount of absorbers to be transported; (ii) traveling to mountains of moderate heights of around $600 \mathrm{~m}$; and (iii) observing significant time-dilation effects from data collected over 5 to $6 \mathrm{~h}$ in a one-day field trip.

The effect of using minimal lead (even as low as no lead at all for the sea level measurements) makes it a variable velocity problem as the muons are slowed down by energy losses in the atmosphere. We use the known expression for the energy loss of the muons in the atmosphere to calculate the velocity profile as the muons travel from the mountain to the sea level and further numerically integrate the varying time-dilation effect to calculate the total travel time of the muons in the Earth frame and in the muon frame.

\section{EXPERIMENTAL SETUP}

The apparatus (shown in Fig. 1) consists of a scintillation detector (a cylinder about 6 in. in diameter and 6 in. high) with a Thorn EMI 9791B photomultiplier held up against the bottom face. Signals from the PMT are sent through a Tennelec 453 discriminator to an EG\&G 466 TAC (time-to-amplitude converter). The TAC registers paired events coming from the muon and the decay electron. The maximum delay time between the muon and the electron pulses is set to $20 \mu$ s on the TAC. This is reasonable considering that the average lifetime of the muon is approximately $2 \mu \mathrm{s}$. Signals from a second detector placed above the muon detector are used to gate the TAC (a feature of the EGG 466) thus processing only those events in which the muon has passed through the upper detector before entering the main detector. The detectors are mounted on separate frames so that the solid angle of acceptance of detectable muons can be varied. A very small acceptance angle limiting detection to the nearly vertical muon flux is desirable to give an easily calculable spectrum, but it results in a very low counting rate and hence a compromise is necessary for optiznal statistics. Our experimental setup accepts flux up to $26^{\circ}$ off-vertical and even with this wide acceptance angle, the difference between the relativistic and nonrelativistic predictions of flux far exceeds the error bars resulting from the varying distances of travel through the atmosphere of the off-vertical flux of muons. This error is at worst comparable with the error resulting from the (Poisson) statistics of counting.

The measurement of the counting rate is made in the 


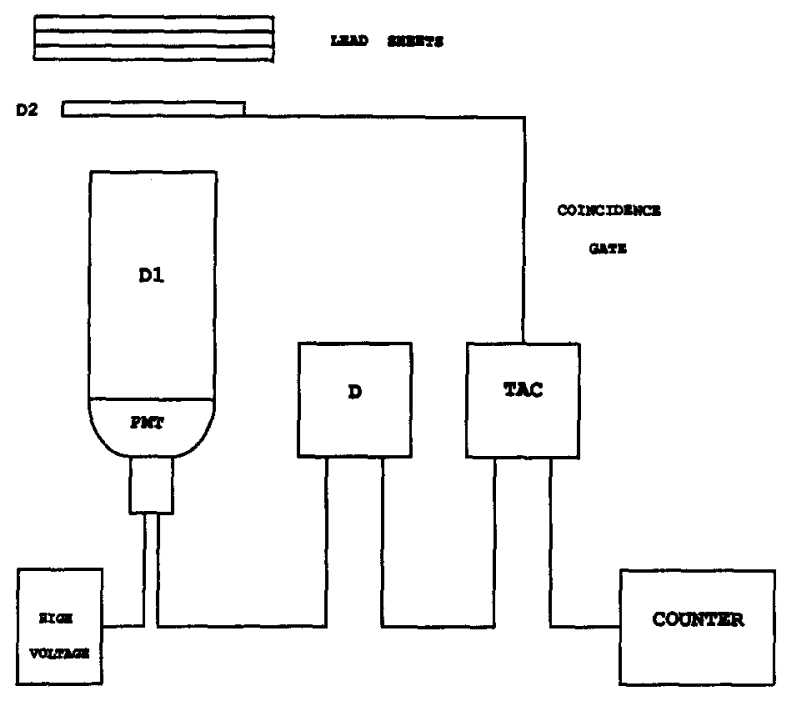

Fig. 1. Schematic diagram of the experimental setup. D1 -the main detector; D2-the detector used in coincidence with D1; PMT-photomultiplier tube; TAC—-time-to-amplitude converter; D-discriminator.

laboratory (sea level) a few days prior to the field trip and the entire setup is transported up to a nearby accessible mountain. Typically a data run of up to 5 or $6 \mathrm{~h}$ is possible in a day's field trip. In order to sample the same band of muon energies on the mountain as those sampled at sea level, it is necessary to introduce the right amount of absorber (lead in our case) above the detectors for the measurements on the mountain. A computer program similar to that described in Sec. III can be used to calculate the thickness of the lead absorbers that would be equivalent to the thickness of the atmosphere between the mountain and sea level ensuring that we are detecting the same sample of muons at both elevations. In order to determine the extent of daily variations of the flux, we monitored the muon flux on several different days over a period of about a month. The daily variations are not large enough to cause concern. Before we discuss the results from our field trip to Marlboro, VT we will describe the numerical calculations necessary to make the theoretical (relativistic and nonrelativistic) predictions of the flux to compare with our measurements.

\section{NUMERICAL CALCULATIONS AND RESULTS}

\section{A. Velocity profile and travel time}

The mean rate of energy loss of the muons as they dissipate energy primarily via interactions with the electrons in the medium is given by

$$
\begin{aligned}
-\frac{d E}{d x}= & \left(\frac{0.307}{\beta^{2}}\right)\left(\frac{Z}{A}\right) \\
& \times\left[\ln \left(\frac{2 m_{0} c^{2}}{I}\right)+\ln \beta^{2}+\ln \gamma^{2}-\beta^{2}\right] \\
& \times \mathrm{MeV} \mathrm{g}^{-1} \mathrm{~cm}^{2},
\end{aligned}
$$

where $\beta=v / c$ is the ratio of the velocity of the muon to that of light, $Z$ and $A$ are atomic number and atomic weight of the absorbing medium, $m_{0}$ is the mass of the electron, and $I$ is the mean excitation energy corresponding to the absorbing medium. ${ }^{3,4}$ Using the specific values of $Z, A$ and
$I^{3}$ for the two media of interest, namely, air (nitrogen) and lead, the above equation becomes

$$
\begin{aligned}
& \left.-\frac{d E}{d x}=\left(\frac{0.1535}{\beta^{2}}\right)\left[9.358+\ln \beta^{2}+\ln \gamma^{2}-\beta^{2}\right)\right] \\
& \quad \times \mathrm{MeV} \mathrm{g}^{-1} \mathrm{~cm}^{2} \\
& \text { for air and } \\
& \qquad \begin{aligned}
-\frac{d E}{d x}= & \left(\frac{0.1216}{\beta^{2}}\right)\left[7.168+\left(\ln \beta^{2}+\ln \gamma^{2}-\beta^{2}\right)\right] \\
& \times \mathrm{MeV} \mathrm{g}^{-1} \mathrm{~cm}^{2}
\end{aligned}
\end{aligned}
$$

for lead. The above functional form has been experimentally verified to within $\pm 1 \% .^{3,4}$ With the above equations, a simple computer program ${ }^{5}$ carries out the following steps of calculation.

(1) For a given input height of the mountain $H$, and an initial value of $\beta$, the program moves the muon through the atmosphere in small steps calculating the energy loss at each step and updating the velocity until it reaches sea level, obtaining a velocity profile $\beta(h)$ of the muon as it travels from the mountain to sea level. The variation of the density of the atmosphere with altitude is also taken into account in the calculation.

(2) The initial value of $\beta=\beta_{i}$ that results in zero velocity at sea level for a given mountain height $H$ is found along with the corresponding profile $\beta(h)$.

(3) For $\beta=\beta_{i}$, the total time elapsed in the Earth frame $\left(t_{E}\right)$ and that in the muon frame $\left(t_{\mu}\right)$ are calculated as

$$
t_{E}=\int_{H}^{0}\left(\frac{1}{c \beta(h)}\right) d h
$$

and

$$
\begin{aligned}
t_{\mu} & =\int_{H}^{0}[c \beta(h) \gamma(h)]^{-1} d h \\
& =\int_{H}^{0}\left(\frac{\left[1-\beta(h)^{2}\right]^{1 / 2}}{c \beta(h)}\right) d h .
\end{aligned}
$$

Figure 2 shows the calculated velocity profile for a muon traveling to sea level from a mountain of height $600 \mathrm{~m}$. Figure 3 is a plot of $t_{E}$ and $t_{\mu}$ for different mountain heights $H$. As expected, the relativistic effects are indeed larger for higher mountains. However, it is interesting to note that

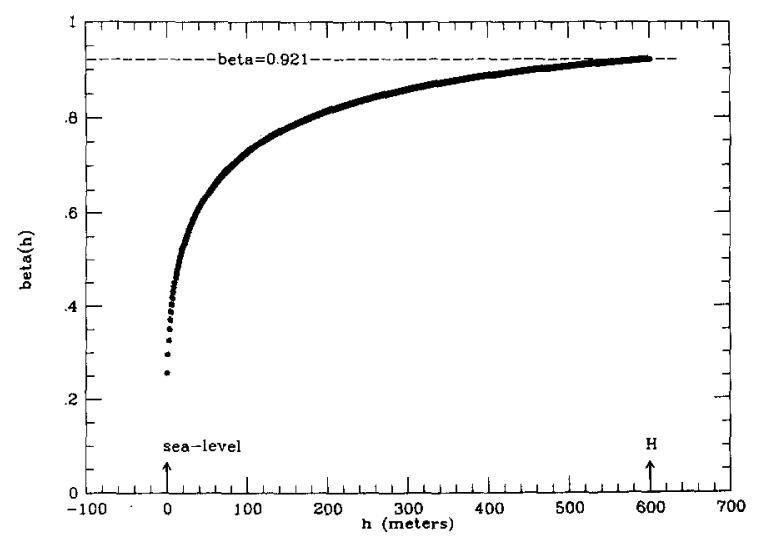

Fig. 2. Calculated velocity profile of a muon traveling from a mountain of height $=600 \mathrm{~m}$ and arriving at sea level with zero velocity. 


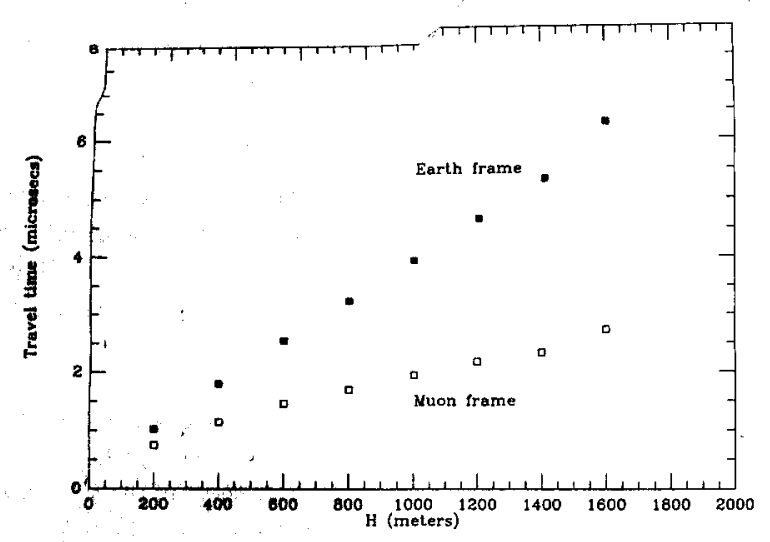

Fig. 3. The calculated travel times $t_{E}$ and $t_{\mu}$ in the Earth and muon frames, respectively, for muons traveling from various mountain heights.

even for a mountain of modest height $=600 \mathrm{~m}, t_{E}$ and $t_{\mu}$ differ by a factor of 1.74. For a given measured flux $N_{s}$ of muons at sea level, the expected flux on the mountain is given by

$$
\begin{aligned}
& N_{r}=N_{s} \exp \left(t_{\mu} / \tau\right), \\
& N_{n r}=N_{s} \exp \left(t_{E} / \tau\right),
\end{aligned}
$$

where $N_{r}$ is from a relativistic calculation and $N_{n r}$ is from a nonrelativistic calculation. Figure 4 is a plot of $N_{r} / N_{r r}$ as a function of mountain height. Note that for a 600 -m mountain $N_{r} / N_{n r}=0.61$. The feasibility of the experiment is hence mainly controlled by the size of the error bars in the measured flux coming from the statistics of the number of counts collected in a typical 5-h data run.

\section{B. Results from the field trip to Marlboro, VT}

Marlboro, VT is at an elevation of $662 \mathrm{~m}$ above our laboratory at Northampton, MA (taken as sea level). The two detectors in coincidence had an acceptance angle of $\sim 26^{\circ}$ at the center of the main detector as mentioned earlier. Prior to the field trip we recorded a muon count of 95 in $5 \mathrm{~h}$ in our laboratory. The thickness of lead required to be transported to the mountain was calculated using the program described earlier to be $11.4 \mathrm{~cm}$. Using $95+10$ (the error bar associated with a total count of $N$ is $N^{1 / 2}$ ) the computer programs described earlier gave the predictions for the counts on the mountain to be $190 \pm 20$ (relativis-

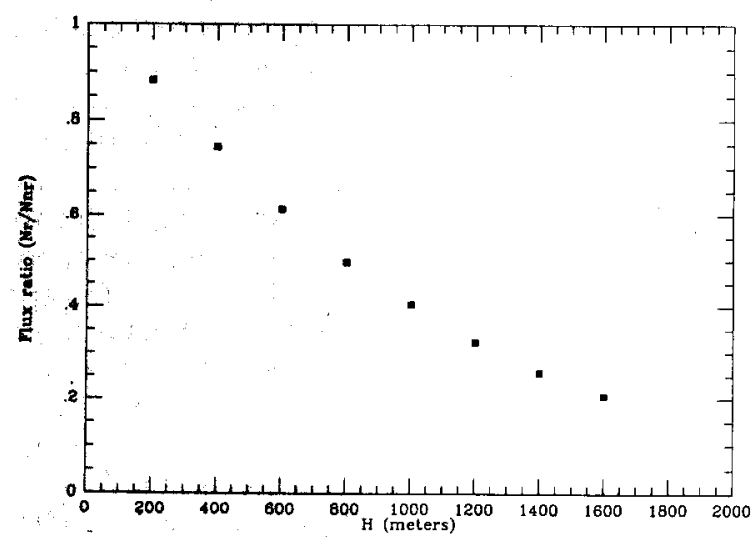

Fig. 4. Ratio of the expected flux from a relativistic calculation to that from a nonrelativistic calculation for various mountain heights. tic) and $330 \pm 60$ (nonrelativistic) for a 5 -h run. On the mountain, the actual muon count was measured to be $110 \pm 11$ in $3 \mathrm{~h}$.

We list below the essential numbers along with the error bars from different sources on the measured data. All the numbers are scaled to a 5- $h$ run for easy comparison: Muon counts at sea level $=95 \pm 10$; predicted counts on the mountain ignoring time dilation $=330 \pm 60$; predicted counts on the mountain taking into account time dilation $=190 \pm 20$; measured counts on the mountain $=183$; error bar in the above measured counts from statistics of counting $= \pm 18$; error bar from the different path lengths of the muon due to finite acceptance angle) $= \pm 8$; error bar in the above measured counts from typical daily variation of flux $= \pm 16$.

The above numbers show a very convincing agreement with the predictions of the relativistic calculation.

\section{USING LEAD AT SEA LEVEL}

If the counting rate from the experimental setup is low or if the height of the mountain is not large, the difference between the relativistic and nonrelativistic predictions may not be significant in comparison with the associated error bars. In such a situation, the feasibility of the experiment could be improved by introducing a small amount of lead over the detector setup at sea level.

It is important to note that the experiment measures the flux of muons that stop in the detector. When there is no lead introduced at sea level, we monitor muons that start with a velocity $\beta_{i}$ on the mountain and end with zero velocity, at sea level. When some amount of lead is introduced for the sea level measurement, the procedure monitors muons that arrive with a nonzero velocity $\beta_{f}$ at sea level (the lead very rapidly slows these to zero velocity as they reach the detector below), thereby scanning muons moving with a larger velocity range $\beta_{i}$ to $\beta_{f}$. In Table I, we show the results of our calculations with different amounts of lead used at sea level $(0$ to $10 \mathrm{~cm})$ for mountains of heights 200 and $400 \mathrm{~m}$. The table lists the calculated total number of muon counts (over a 5-h data run) on the mountain for a sea level count rate of 100 muons over $5 \mathrm{~h}$ that we typically measure with our coincidence setup. This helps the interpretation of the table in the light of the error bars associated with the statistics of particle counting. Clearly, a mountain of height $200 \mathrm{~m}$ is too small to perform a statistically signif-

Table I. The calculated total number of counts (for a 5-h data run) on the mountain for different thicknesses of lead used at sea level, scaled for a typical sea level count rate of 100 muons in $5 \mathrm{~h}$.

\begin{tabular}{cccc}
\hline \hline $\begin{array}{c}\text { Height of } \\
\text { mountain } \\
(\mathrm{m})\end{array}$ & $\begin{array}{c}\text { Thickness of } \\
\text { lead at sea } \\
\text { level }(\mathrm{m})\end{array}$ & $\begin{array}{c}\text { Expected counts from calculations } \\
\text { (Relativistic) }\end{array}$ & (Nonrelativistic) \\
\hline \multirow{2}{*}{200} & 0 & 141 & 159 \\
& 2 & 123 & 144 \\
& 6 & 116 & 140 \\
& 10 & 112 & 138 \\
400 & 0 & 169 & 226 \\
& 2 & 145 & 204 \\
& 6 & 130 & 194 \\
& 10 & 124 & 190 \\
\hline \hline
\end{tabular}


icant experiment unless the data collection time or the count rate with the setup is higher. The introduction of lead at sea level increases the differences between the relativistic and nonrelativistic predictions for the muon count on the mountain. However, excessive lead introduced at sea level makes the travel times too small in the case of low mountains, bringing the expected counts on the mountain closer to those at sea level. Clearly, a proper compromise of the parameters can be made to suit the height of the mountain and the counting rate from the setup.

\section{CONCLUSIONS}

A significant experiment can be performed with a daylong field trip to a moderately high nearby mountain. The concept of relativistic time dilation is both exciting and difficult for a student in the first modern physics course and this experiment helps to convert skeptics to believers. Clearly, the pedagogical value of such an experiment is immense, besides, of course, the experience that students gain in working with modern equipment and techniques of particle detection.

\section{ACKNOWLEDGMENTS}

The equipment for particle detection was bought with funding from the NSF-CSIP program (Award \# CSI-8650414) with matching funds from Smith College, Northampton, MA. We also acknowledge Elizabeth Karagosian for her help with the software.

\footnotetext{
a) Present address: University of California at Santa Cruz, CA 95064.

'B. Rossi, Cosmic Rays (McGraw-Hill, New York, 1964), Chap. 8.

${ }^{2}$ David H. Frisch and James H. Smith, "Measurement of the relativistic time dilation using $\mu$-mesons," Am. J. Phys. 31, 342-355 (1963).

${ }^{3} \mathrm{U}$. Fano, "Penetration of protons, alpha particles and mesons," Ann. Rev. Nucl. Sci. 13, 1-66 (1963).

4'Particle Data Group, "Review of particle properties," Rev. Mod. Phys. 52, S44-S46 (1980) and Phys. Lett. B 204, 64-68 (1988).

${ }^{5}$ The software is written using Microsoft Quick BASIC for IBM-compatible personal computers and is available on request.
}

\title{
The two-slit interferometer reexamined
}

\author{
E. C. G. Sudarshan and Tony Rothman ${ }^{\text {a) }}$ \\ Center for Particle Theory, University of Texas, Austin, Texas 78712
}

(Received 2 April 1990; accepted for publication 9 October 1990)

It is shown that the standard exposition of the two-slit diffraction experiment is incorrect because it treats the interference as arising from the photon wave function $\psi$, whereas the interference is really between coherent states of the field, which do not correspond to single-photon states. Several other misconceptions are pointed out.

\section{INTRODUCTION}

The two-slit interferometer, or two-slit diffraction experiment as it is called equally often, stands at the center of the conceptual foundations of quantum mechanics. Such is the importance of the two-slit experiment that discussions of its paradoxical nature are invariably found in every introductory quantum mechanics text. With hundreds of expositions, familiar to every student and instructor of quantum mechanics, one might reasonably suppose that nothing more can be said about the subject. In fact, this is far from the case. A close examination of the standard exposition reveals several conceptual errors and ambiguities, principally due to a confusion between the electromagnetic field amplitude and the amplitude of the quantum mechanical wave function. A correct treatment of the two-slit experiment necessarily requires a field-theoretic approach. In this note we outline such a treatment and show in what limits the textbook discussions correspond to it.

\section{PROBLEMS}

To begin the discussion we cite several examples-taken somewhat at random from available textbooks - that illustrate the difficulties with the standard approach. The first passage is from Merzbacher, ${ }^{1}$
In physical optics interference patterns are produced by the superposition of waves of $\mathbf{E}$ and $\mathbf{H}$ but the intensity of the fringes is measured by $\mathbf{E}^{2}$ and $\mathbf{H}^{2}$. In analogy to this situation we assume that the positive quantity $|\psi(x, y, z, t)|^{2}$ measures the probability of finding a particle at $x, y, z$.

\section{In Park's text, ${ }^{2}$ we find}

The particle picture is of no help here, for it makes sense to talk of the intensity of a beam of particles but not its amplitude, and the construction of the interference pattern explicitly requires the superposition of the amplitudes of the component waves. A further indication of the short comings of this picture is given by experiments on interference between light beams which are so faint that, in the particle picture, there would almost never to be two particles inside the apparatus at the same time. A particle, if such there were, would go through only one slit. Thus the existence of the other slit would make no difference and an incoherent superposition would result. In fact, however, when a long exposure is made, ordinary wave interference fringes are produced, the conflict between the wave and particle picture being made especially striking by the fact that, as always, the dark- 